\title{
Theoretical Modelling of High-efficiency Perovskite Solar Cells and Reduction of Internal Heat Generation using Hot-Electron Extraction
}

\author{
Ali Rostami ( $\sim$ rostami@tabrizu.ac.ir) \\ University of Tabriz https://orcid.org/0000-0002-8727-4711 \\ Isun Tofigi \\ University of Tabriz
}

Azeez Barzinjy

Salahaddin University - Erbil College of Education

Hamit Mirtagioglu

Bitlis Eren Universitesi

\section{Research Article}

Keywords: Methylammonium lead halide, perovskite material, selective energy contacts, solar cell, internal heat reduction, stability, long lifetime.

Posted Date: August 13th, 2021

DOl: https://doi.org/10.21203/rs.3.rs-791363/v1

License: (9) This work is licensed under a Creative Commons Attribution 4.0 International License. Read Full License 


\title{
Theoretical Modelling of High-efficiency Perovskite Solar Cells and Reduction of Internal Heat Generation using Hot-Electron Extraction
}

\author{
A. Rostami ${ }^{1,2}$, I. Tofigi ${ }^{1}$, Azeez A. Barzinjy ${ }^{3,4}$, and H. Mirtagioglu ${ }^{5}$ \\ 1 Photonics and Nanocrystals Research Lab (PNRL), University of Tabriz, Tabriz, Iran \\ ${ }^{2}$ SP-EPT Lab, ASEPE Company, Industrial Park of Advanced Technologies, Tabriz, 5364196795, Iran \\ ${ }^{3}$ Department of Physics, College of Education, Salahaddin University-Erbil, Iraq \\ ${ }^{4}$ Physics Education Department, Education Faculty, Tishk International University, Erbil, Iraq \\ ${ }^{5}$ Department of Statistics, Faculty of Science and Literature, University of Bitlis Eren, Bitlis, Turkey
}

\begin{abstract}
Perovskite single crystals have received enormous attention in recent years. This is, perhaps, due to their simplistic synthesis and excellent optoelectronic properties including the long carrier diffusion length, high carrier mobility, low trap density, and tuneable absorption edge ranging from ultra-violet (UV) to near-infrared (NIR). These distinguishing features offer numerous potential applications in energy-related fields like solar cells, photodetectors (PDs), lasers, etc. Efficiency enhancement and stability, in general, are the main challenges to obtain better solar cells. One of the main reasons for the early degradation of solar cells is heat generation due to high energy electrons and holes in the conduction and valance bands. In this study, the authors trying to introduce the concept of selective energy contacts in perovskite solar cells. Also, they investigate how this concept affects the power conversion efficiency (enhancement) and heat generation due to hot electrons and holes (reduction) scattering in the conduction and valance bands. Both efficiency enhancement and reduction in heat generation have been calculated in this study. Thus, for mathematical modeling of the anticipated idea, the Methylammonium lead halide $\left(\mathrm{CH}_{3} \mathrm{NH}_{3} \mathrm{Pbl}_{3}\right)$ material is used in a PIN structure for a single-junction solar cell. Also, for the proposed structure, analytical modeling was introduced and it is shown that the efficiency of a single contact cell is around $25 \%$, and after applying the second contact, the efficiency was increased to $35 \%$. Finally, due to the reduction of heat loss in the structure, the stability of perovskite material is significantly increased.
\end{abstract}

Keywords: Methylammonium lead halide; perovskite material; selective energy contacts; solar cell; internal heat reduction; stability; long lifetime.

\section{Introduction}

Nowadays, the energy required by humans is provided by renewable and non-renewable sources. Renewable energy resources have received more attention due to the lack of traditional resources such as fossil fuels. Solar cells are among the most important devices to convert solar energy as a renewable energy resource and today extensive research is being done in this field [1-5]. In the past decade and with the advent of the third generation of solar cells, the use of perovskite in solar cells has received a lot of attention in the industry due to its many distinctive properties $[6,7]$. Characteristics such as the high absorption coefficient, high carrier mobility, long carrier diffusion length, suitable bandgap, and ease of fabrication of this material, which make the cost of constructing a solar cell very low, have caused this material to be used more than any other material in making solar cells $[8,9,10]$. 
Perovskite solar cells were first introduced in 2009 with only 3.8\% efficiency [11]. According to the latest reports in 2019 , scientists were able to achieve an efficiency of $25.5 \%$ in the P-I-N structure solar cell [12]. This is an incredible achievement in increasing efficiency within a very short period. Nonetheless, perovskite material posed many challenges to the solar cell industry. The structure of perovskite is $A B X_{3}$ where $A$ is $\mathrm{CH}_{3} \mathrm{NH}_{3}+\mathrm{HC}\left(\mathrm{NH}_{2}\right)_{2}+$ or $\mathrm{Cs}+, \mathrm{B}$ is $\mathrm{Pb}_{2}+$ or $\mathrm{Sn}_{2}+$ and $X$ is one of Halogen elements $(\mathrm{I}-/ \mathrm{Br}-/ \mathrm{Cl}-)(13,14,15]$. The most common material of the perovskite family, which can be used in the construction of solar cells due to possessing a suitable energy gap of 1.55 eV is methylammonium lead iodide $\left(\mathrm{CH}_{3} \mathrm{NH}_{3} \mathrm{Pbl}_{3}\right)[16]$.

Currently, research in the field of perovskite solar cells is mainly focused on increasing the efficiency and stability of perovskite material. The main problem and disadvantage of perovskite material is its instability. Perovskite solar cells are highly unstable to heat, oxygen, UV rays, and other factors and over time, it disintegrates and loses its original properties, which reduces the initial efficiency of the solar cell [17]. Therefore, the most important challenge in perovskite solar cells is to increase the efficiency and also increase perovskite stability under the above factors. Recently, massive efforts have been made to study the degradation mechanism and stability enhancement strategies. Much research has been done in recent years about increasing perovskite solar cell efficiency and increasing the stability of perovskite material under the influence of various factors. Research teams in the field of solar cells, by adjusting the parameters of the perovskite solar cell, such as the values of layer thicknesses, doping concentrations, and energy ranges of the PIN junction solar cell were able to obtain the all-perovskite solar cell with high power and conversion efficiency of $25.84 \%$. [17, 18]. This achievement is highly appreciated, however, the issue in these attempts did not pay any attention to the factors that reduce the stability of this material. This issue significantly reduces efficiency over a short period [19]. Also, reports based on other studies using the adjustable bandwidth property and soft processing temperature of perovskite solar cells in tandem solar cells show $25 \%$ efficiency [20]. Then again the efficiency is still lower compared to other materials such as silicon. In other investigations, by changing the chemical formula of the substance or through doping cesium, they could increase the thermal stability to some extent [21]. In other words, Cs doping enhanced the device's performance and perovskite stability. However, since the impurity activation in the perovskite changes other attributes so it does not appear to be a good choice [21].

In fact, in most of the studies carried out so far to increase the stability of perovskite under various factors, the focus has been on the change in the composition and chemical nature of the material, and no attention has been paid to the factors that affect the overall nature of the cell structure, such as losses in solar cells. In general, various factors reduce the efficiency of the solar cell, while the most important one is the losses in the solar cell. Various factors affecting the losses in the solar cell, but the most significant one is the hot carriers. Indeed, some carriers cannot be absorbed and converted into electricity by bonding since their energy is greater than the energy gap of the utilized matter, therefore they form hot carriers. Accordingly, before absorption, they disappear and appear as a phonon, and this factor causes the solar cell to heat up and also reduces the efficiency of the whole cell. Therefore, one should provide a solution, by eliminating these losses, which lead to increasing the efficiency of the solar cell, 
preventing the cell temperature from rising through the production of phonon, and increasing the thermal stability of perovskite material. Consequently, the authors of this investigation proposed a theory of energy-selective contacts, which is designed at the PIN junction for the first time, includes three parts of the $\mathrm{CH}_{3} \mathrm{NH}_{3} \mathrm{Pbl}_{3}$ material. With these contacts that are connected to the solar cell substrate, the carriers can be absorbed with whatever amount of energy they have and prevent the solar cell from losing heat, reducing the efficiency and stability of the material. In this study, using the selective energy contacts high-efficiency perovskite solar cell is introduced and the reduction of internally generated heat is calculated. It is shown that with increasing the number of contacts, the heat generation is decreased besides stability and efficiency are enhanced.

In section II, the structure and operation principles are presented. Also, mathematical modeling of selective contacts and other parameters of the perovskite solar cells are presented in section III. Simulation results are illustrated and discussed in section IV.

\section{Structure and operation principles}

To increase the efficiency of the solar cell, the authors of the current study use the selective energy contacts that are used on the PIN junction as shown in Fig. 1. In the schematic, the second contact has energy higher than the edge of the conduction band and collects high-energy electrons.

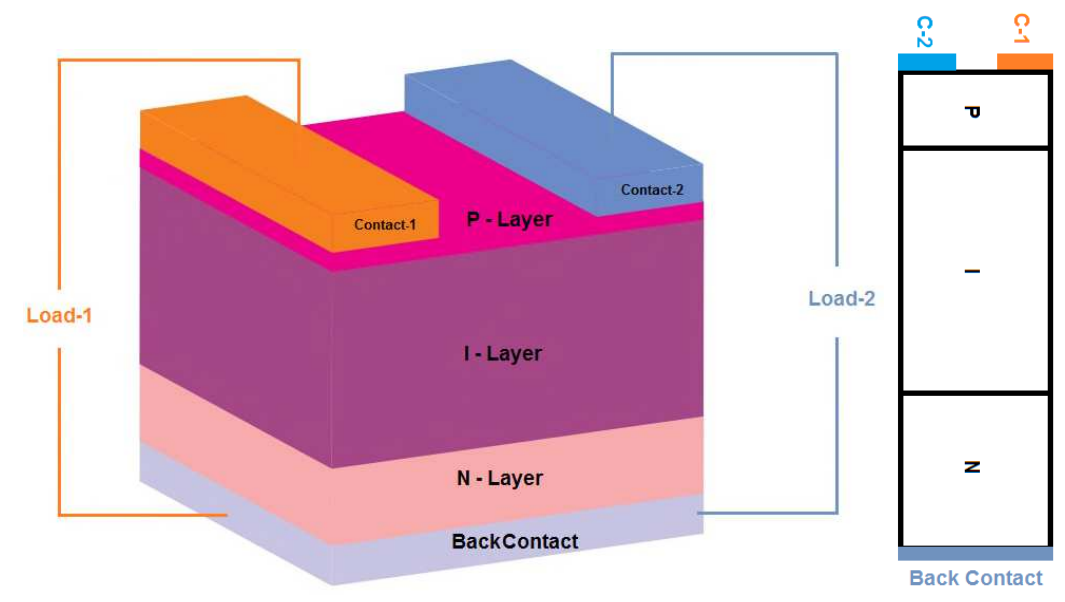

Fig. 1. Schematic diagram of the pin-type all perovskite solar cell

All parameters required in the numerical simulation are given in Table 1.

Table 1. Simulation parameters of perovskite solar cells in this study

\begin{tabular}{|l|c|c|c|}
\hline \multicolumn{1}{|c|}{ Parameters } & $\mathrm{N}$ - Layer & $\mathrm{I}-$ Layer & $\mathrm{P}$ - Layer \\
\hline Material & $\mathrm{CH}_{3} \mathrm{NH}_{3} \mathrm{Pbl}_{3}$ & $\mathrm{CH}_{3} \mathrm{NH}_{3} \mathrm{Pbl}_{3}$ & $\mathrm{CH}_{3} \mathrm{NH}_{3} \mathrm{Pbl}_{3}$ \\
\hline Thickness $(\mathrm{nm})$ & 100 & 300 & 10 \\
\hline Eg $(\mathrm{eV})$ & 1.55 & 1.55 & 1.55 \\
\hline Relative Permittivity $\left(\varepsilon_{r}\right)$ & 6.5 & 6.5 & 6.5 \\
\hline Donor doping concentration (ND 1/cm $\left.{ }^{3}\right)$ & $10^{18}$ & $10^{13}$ & 0 \\
\hline Acceptor doping concentration (ND 1/cm $\left.{ }^{3}\right)$ & 0 & 0 & $10^{18}$ \\
\hline
\end{tabular}


In Fig. 1 a PIN structure is demonstrated and all three regions consist of the same perovskite substance of the methylammonium lead-iodide type with the chemical formula $\mathrm{CH}_{3} \mathrm{NH}_{3} \mathrm{Pbl}_{3}$. The absorption region or I-layer is sandwiched between two layers $\mathrm{N}$ and $\mathrm{P}$. The $\mathrm{N}$ and $\mathrm{P}$ semiconductor layers are doped with acceptor and donor atoms with a certain amount of doping, however, the absorption layer is very low, and sometimes approaches zero. The operation of this structure is similar to the PN junction, except that there is no drift current in the PN junction, but in this structure, due to the presence of the absorption region and the discharge region, there are both components of the drift and diffusion currents. In a simple solar cell, one can use a p-n or p-i-n structure to separate the charge carriers generated by photon absorption. In such structures, electrons and holes migrate from high-concentration regions to low-concentration regions, resulting in diffusion currents. Likewise, an electric field in the intrinsic region makes drift currents as well. In the following, initially, the theory of selective energy contacts is presented. There are two contacts on the top of this structure, one back contact on the $\mathrm{n}$ side and two contacts on the $\mathrm{p}$ side, each of which is connected to the back contact via a separate load. Sunlight also enters the solar cell from the p-region. To realize the theory of contacts with selective energy, we have a back contact on the $\mathrm{n}$ side of the cell to collect the total current of the holes, and on the $\mathrm{p}$ side, where sunlight is propagated, we have two separate contacts to collects electrons with different energies. It is located on the top side of the PIN cell. In the solar cell, the total flow of electrons can be considered. These contacts are arranged to extract the generated carriers with two ranges of low and high energies. The energy of the first contact is close to the conduction band, and the energy difference between contacts is equal to $\Delta \mathrm{E}$. The figure below (Fig. 2) shows the solar spectrum and absorption of the perovskite material and the energy band diagram. Also, it shows the position of the contacts and the distribution of electrons. As shown in Fig. 2, through the two contacts defined, the generated carriers are divided into two parts based on their energy. In this solar spectrum, the high-energy region is associated with the second contact, and the low-energy region is linked to the first contact. With two separate energy ranges, the contacts collect electrons at different energy levels, meaning that the concentration of electrons is divided between these contacts and then extracted by them separately. In this way, each of them possesses a separate output. The second contact, which has a higher energy level than the first contact, extracts higher energy carriers according to their energy range. Thus the residual carriers are in the same energy range of the first contact and they are extracted through this contact.
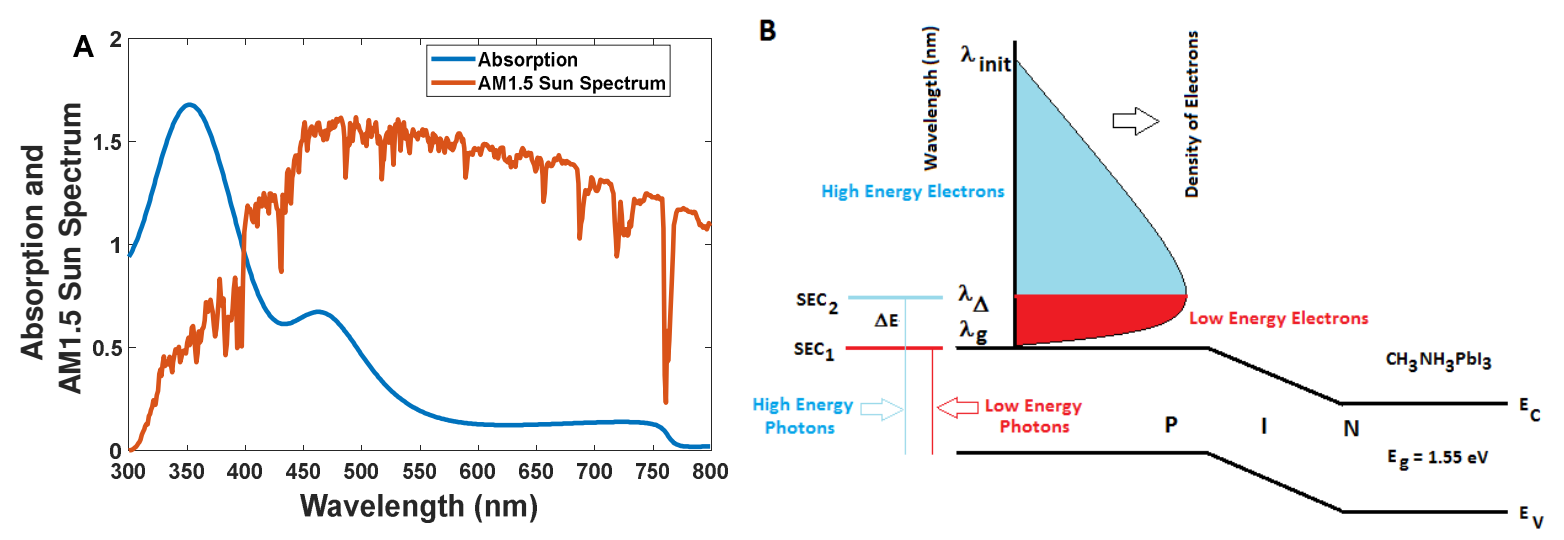
Fig. 2. A) AM1.5 Sun spectrum $\left(1000 \mathrm{~W} / \mathrm{m}^{2}\right)$ and spectral absorption of $\mathrm{CH}_{3} \mathrm{NH}_{3} \mathrm{Pbl}$, B) Energy Band Diagram and two selective energy contacts,

As a result, despite the many causes of losses in solar cells, we show that by absorbing more energy carriers from the physical bandgap, these losses can be reduced and the cell efficiency increased at the same time. To calculate the electric power captured by each contact, we examine the wavelength range of the solar spectrum that can be absorbed, and accordingly, the electron-hole pairs are generated by each contact. In this calculation, AM1.5 for an incident solar pattern is considered. In Eq. 1, energy and wavelength relation is introduced to figure out the absorbed solar spectrum energy to generate electron-hole pairs.

$E=\frac{h c}{\lambda}$

where $h$ is Planck's constant, $\mathrm{c}$ is the speed of light and $\lambda$ is the wavelength. As we know, the absorption wavelength range of perovskite is proportional to its bandwidth energy from $300 \mathrm{~nm}$ to $800 \mathrm{~nm}$.

\section{Mathematical Modeling of PIN Solar Cell with two Selective Energy Contacts (SECs)}

To begin, we solve the continuity equations for the three regions of the cell. For the $\mathrm{P}$ and $\mathrm{N}$ regions, which are the quasi-neutral regions of the cell, the predominant current is the diffusion current, and in layer I, which is assumed to be completely discharged, the predominant current is the drift current. By solving these coupling equations for the P and $\mathrm{N}$ regions, we extract the electrons and holes from the diffusion current, and by solving these equations again for the discharge region, we obtain the drift current for the absorption layer I.

$\frac{\partial \Delta n}{\partial t}=-\frac{1}{q} \frac{\partial J_{n}}{\partial x}+\left(G_{n}-R_{n}\right)$

$\frac{\partial \Delta p}{\partial t}=\frac{1}{q} \frac{\partial J_{p}}{\partial x}+\left(G_{p}-R_{p}\right)$

where $\Delta n, \Delta p, J_{n}, J_{p}, G_{n}$ and $G_{p}$ are excess minority carriers of electrons in the P semiconductor layer, excess hole carriers in the $\mathrm{N}$ layer, electron current density, hole current density, electron generation rate, and hole generation rate respectively. In this work, only the Shockley-Reed-Hall recombination process is considered. For incoming light into the solar cell at depth $\mathrm{X}$, the generation rate is given as follows.

$G_{n, p}(x)=\int_{\lambda: \lambda_{\text {init }}-\lambda_{g}} f(\lambda) \cdot \alpha(\lambda) \cdot \mathrm{e}^{\left(-\alpha(\lambda)\left(x+w_{p}\right)\right)} d \lambda$

where $f(\lambda)$ is the photon flux of the incoming light and $\alpha(\lambda)$ is the absorption coefficient of the material, which is considered for the perovskite material used in the solar cell. Also, the range of wavelength that integration is doing on is determined by sunlight and material bandgap. Now, using Eqs. 2, 3, and considering the diffusion component of the 
current density, the following differential equations for electrons and holes in quasi-neutral regions of $\mathrm{P}$ and $\mathrm{N}$ layers can be obtained.

$$
\begin{gathered}
D_{p} \frac{d^{2} \Delta p}{d x^{2}}-\frac{\Delta p}{\tau_{p}}=-G_{p}(x) \\
D_{n} \frac{d^{2} \Delta n}{d x^{2}}-\frac{\Delta n}{\tau_{n}}=-G_{n}(x)
\end{gathered}
$$

where $D_{p}, D_{n}, \tau_{p}$ and $\tau_{n}$ are diffusion coefficient for holes, the diffusion coefficient for electrons, hole and electron relaxation times (mean free times) respectively. Also, $G_{n}(x)$ and $G_{p}(x)$ are the generation rates for electrons and holes correspondingly. Now, considering Eq. 5 the excess hole carrier transport in the $\mathrm{N}$ layer can be obtained as follows.

$$
\Delta p_{n}(x)=A_{n} \sinh \left(\frac{x-x_{n}}{L_{p}}\right)+B_{n} \cosh \left(\frac{x-x_{n}}{L_{p}}\right)+\Delta p_{n}\left(G_{p}(x)\right)
$$

where $\Delta p_{n}\left(G_{p}(x)\right)$ is the special solution of excess hole carriers due to the generation rate made by sunlight irradiation and can be calculated as follows.

$\Delta p_{n}\left(G_{p}(x)\right)=\zeta \int_{\lambda: \lambda_{\text {init }}-\lambda_{g}} f(\lambda) \cdot \alpha(\lambda) \cdot \mathrm{e}^{\left(-\alpha(\lambda)\left(x+w_{p}\right)\right)} d \lambda$

where $\zeta=-\frac{\tau_{p}}{L_{p}^{2} \alpha^{2}(\lambda)-1}$ and $L_{p}=\sqrt{D_{p} \tau_{p}}$ that is mean free path for holes. Now, it is necessary to consider the boundary conditions to obtain $A_{n}$ and $B_{n}$. For boundary conditions, we consider excess hole carriers and slope of that at $\mathrm{x}=\mathrm{x}_{\mathrm{n}}$ and $\mathrm{x}=\mathrm{W}_{\mathrm{n}}$ respectively. In this work, we assume that all holes are collected and managed by the first contact.

$\Delta p_{n}\left(x=x_{n}\right)=p_{n_{0}}\left(e^{\frac{V_{1}}{V_{T}}}-1\right)$

where $\mathrm{V}_{1}, \mathrm{~V}_{\mathrm{T}}$, and $\mathrm{P}_{\mathrm{n} 0}$ are electric potential at first contact, thermal voltage, and $p_{n_{0}}=\int_{-\infty}^{E_{V}} D(E) \cdot F_{p}(E) d E$ respectively. Also, the rate of recombination of holes at the end of the $\mathrm{N}$ layer is considered as follows.

$\left.\frac{\mathrm{d} \Delta \mathrm{p}}{\mathrm{dx}}\right|_{x=W_{n}}=\frac{\mathrm{S}_{\mathrm{P}}}{\mathrm{D}_{\mathrm{p}}} \Delta \mathrm{p}_{n}\left(\mathrm{w}_{\mathrm{n}}\right)$

where $S_{p}$ is surface recombination rate. By applying the boundary conditions, the constants $A_{n}$ and $B_{n}$ are calculated as follows. 


$$
\begin{aligned}
& A_{n}=\left[\frac{\frac{D_{p}}{L_{p}} \sinh \left(\frac{W_{n}-x_{n}}{L_{p}}\right)-S_{P} \cosh \left(\frac{W_{n}-x_{n}}{L_{p}}\right)}{S_{P} \sinh \left(\frac{W_{n}-x_{n}}{L_{p}}\right)-\frac{D_{p}}{L_{p}} \cosh \left(\frac{W_{n}-x_{n}}{L_{p}}\right)}\right]\left[p_{n_{0}}\left(e^{\frac{V_{1}}{V_{T}}}-1\right)-\Delta p_{n}\left(G_{p}\left(x_{n}\right)\right)\right]+ \\
& {\left[\frac{D_{p} \frac{d \Delta p_{n}\left(G_{p}\left(W_{n}\right)\right)}{d x}-S_{P} \Delta p_{n}\left(G_{p}\left(W_{n}\right)\right)}{S_{P} \sinh \left(\frac{W_{n}-x_{n}}{L_{p}}\right)-\frac{D_{p}}{L_{p}} \cosh \left(\frac{W_{n}-x_{n}}{L_{p}}\right)}\right]} \\
& B_{n}=p_{n_{0}}\left(e^{\frac{V_{1}}{V_{T}}}-1\right)+\int_{\lambda_{\text {init }}}^{\lambda_{g}} \frac{\tau_{p}}{L_{p}^{2} \alpha^{2}(\lambda)-1} f(\lambda) \alpha(\lambda) e^{-\alpha(\lambda)\left(x_{n}+W_{p}\right)} d \lambda
\end{aligned}
$$

Similar to the procedure for excess hole carriers, one can do for excess electron carriers as follows. It should be mentioned that in the conduction band there are two contacts with excess electrons named $\Delta n_{1}$ and $\Delta n_{2}$ that associated with electrons having energy in the interval $\lambda_{\Delta}-\lambda_{g}$ and $\lambda_{g}-\lambda_{\text {init }}$ respectively.

$\Delta n_{p_{1}}(x)=A_{p_{1}} \sinh \left(\frac{x-x_{p}}{L_{n}}\right)+B_{p_{1}} \cosh \left(\frac{x-x_{p}}{L_{n}}\right)+\Delta n_{p_{1}}\left(G_{n}(x)\right)$

where $\Delta n_{p_{1}}\left(G_{n}(x)\right)=\int_{\lambda_{\Delta}}^{\lambda_{g}}-\frac{\tau_{n}}{L^{2}{ }_{n} \alpha^{2}(\lambda)-1} f(\lambda) \cdot \alpha(\lambda) \cdot \mathrm{e}^{\left(-\alpha(\lambda)\left(x+w_{p}\right)\right)} d \lambda$

$\Delta n_{p_{2}}(x)=A_{p_{2}} \sinh \left(\frac{x-x_{p}}{L_{n}}\right)+B_{p_{2}} \cosh \left(\frac{x-x_{p}}{L_{n}}\right)+\Delta n_{p_{2}}\left(G_{n}(x)\right)$

where $\Delta n_{p_{2}}\left(G_{n}(x)\right)=\int_{\lambda_{\text {init }}}^{\lambda_{\Delta}}-\frac{\tau_{n}}{L^{2}{ }_{n} \alpha^{2}(\lambda)-1} f(\lambda) \cdot \alpha(\lambda) \cdot \mathrm{e}^{\left(-\alpha(\lambda)\left(x+w_{p}\right)\right)} d \lambda$

where $L_{n}=\sqrt{D_{n} \tau_{n}}$ is the mean free path for electron. Now, it is necessary to consider the boundary conditions to obtain the $A_{p_{1,2}}$, and $B_{p_{1,2}}$. For boundary conditions, we consider excess electron carrier and slope of that at $X=-X_{p}$ and $X=-W_{p}$ respectively.

$\Delta n_{p_{1}}\left(x=-x_{p}\right)=n_{p_{0_{1}}}\left(e^{\frac{V_{1}}{V_{T}}}-1\right)$

where $n_{p_{0_{1}}}=\int_{E_{C}}^{E_{C}+\Delta E} D(E) \cdot F_{n}(E) d E, \mathrm{~V}_{1}, \mathrm{~V}_{\mathrm{T}}$, and $\mathrm{n}_{001}$ are electric potentials at first contact, thermal voltage, and density of electrons around the first contact respectively. 
$\Delta n_{p_{2}}\left(x=-x_{p}\right)=n_{p_{0_{2}}}\left(e^{\frac{V_{2}}{V_{T}}}-1\right)$

where $n_{p_{02}}=\int_{E_{C}+\Delta E}^{+\infty} D(E) \cdot F_{n}(E) d E \mathrm{~V}_{2}$ and $\mathrm{n}_{\mathrm{p} 02}$ are voltage on second contact and density of electrons above the second contact. Also, the rate of recombination of electrons at the end of the P region is considered as follows.

$\left.\frac{\mathrm{d} \Delta \mathrm{n}_{p_{1}}}{\mathrm{dx}}\right|_{x=-W_{p}}=\frac{\mathrm{S}_{\mathrm{n}}}{\mathrm{D}_{\mathrm{n}}} \Delta \mathrm{n}_{p_{1}}\left(-\mathrm{W}_{\mathrm{p}}\right)$
$\left.\frac{\mathrm{d} \Delta \mathrm{n}_{p_{2}}}{\mathrm{dx}}\right|_{x=-W_{p}}=\frac{\mathrm{S}_{\mathrm{n}}}{\mathrm{D}_{\mathrm{n}}} \Delta \mathrm{n}_{p_{2}}\left(-\mathrm{W}_{\mathrm{p}}\right)$

where $S_{n}$ and $D_{n}$ are surface recombination and diffusion coefficient of electrons in the $P$ region. Now using the boundary conditions the constants in Eqs. 13 and 14 can be calculated as follows.

$$
\begin{aligned}
& A_{p_{1}}=\left[\frac{\frac{D_{n}}{L_{n}} \sinh \left(\frac{-W_{p}+X_{p}}{L_{n}}\right)-S_{n} \cosh \left(\frac{-W_{p}+X_{p}}{L_{n}}\right)}{S_{n} \sinh \left(\frac{-W_{p}+X_{p}}{L_{n}}\right)-\frac{D_{n}}{L_{n}} \cosh \left(\frac{-W_{p}+X_{p}}{L_{n}}\right)}\right]\left[n_{p_{0_{1}}}\left(e^{\frac{V_{1}}{V_{T}}}-1\right)-\Delta n_{p_{1}}\left(G_{n}\left(-X_{p}\right)\right)\right]+ \\
& {\left[\frac{D_{n} \frac{d \Delta n_{p_{1}}\left(G_{n}\left(-W_{p}\right)\right)}{d x}-S_{n} \Delta n_{p_{1}}\left(G_{n}\left(-W_{p}\right)\right)}{S_{n} \sinh \left(\frac{-W_{p}+X_{p}}{L_{n}}\right)-\frac{D_{n}}{L_{n}} \cosh \left(\frac{-W_{p}+X_{p}}{L_{n}}\right)}\right]} \\
& B_{p_{1}}=n_{p_{01}}\left(e^{\frac{V_{1}}{V_{T}}}-1\right)+\int_{\lambda_{\Delta}}^{\lambda_{g}} \frac{\tau_{n}}{L_{n}^{2} \alpha^{2}(\lambda)-1} f(\lambda) \cdot \alpha(\lambda) e^{-\alpha(\lambda)\left(W_{p}-X_{p}\right)} d \lambda
\end{aligned}
$$

Similarly, we have

$$
\begin{aligned}
A_{p_{2}}= & {\left[\frac{\frac{D_{n}}{L_{n}} \sinh \left(\frac{-W_{p}+X_{p}}{L_{n}}\right)-S_{n} \cosh \left(\frac{-W_{p}+X_{p}}{L_{n}}\right)}{S_{n} \sinh \left(\frac{-W_{p}+X_{p}}{L_{n}}\right)-\frac{D_{n}}{L_{n}} \cosh \left(\frac{-W_{p}+X_{p}}{L_{n}}\right)}\right]\left[n_{p_{0_{2}}}\left(e^{\frac{V_{2}}{V_{T}}}-1\right)-\Delta n_{p_{2}}\left(G_{n}\left(-X_{p}\right)\right)\right]+} \\
& {\left[\frac{D_{n} \frac{d \Delta n_{p_{1}}\left(G_{n}\left(-W_{p}\right)\right)}{d x}-S_{n} \Delta n_{p_{1}}\left(G_{n}\left(-W_{p}\right)\right)}{S_{n} \sinh \left(\frac{-W_{p}+X_{p}}{L_{n}}\right)-\frac{D_{n}}{L_{n}} \cosh \left(\frac{-W_{p}+X_{p}}{L_{n}}\right)}\right] }
\end{aligned}
$$


$B_{p_{2}}=n_{p_{0_{2}}}\left(e^{\frac{V_{2}}{V_{T}}}-1\right)+\int_{\lambda_{\text {init }}}^{\lambda_{\Delta}} \frac{\tau_{n}}{L_{n}^{2} \alpha^{2}(\lambda)-1} f(\lambda) \cdot \alpha(\lambda) e^{-\alpha(\lambda)\left(W_{p}-X_{p}\right)} d \lambda$

Now, using minority charge carriers distribution, the current density can be obtained for the solar cell. The current density includes minority hole carriers in the $\mathrm{N}$ region, minority electron carriers in the $\mathrm{P}$ region, and generation and recombination current in the intrinsic area. Minority hole current density in the $\mathrm{N}$ region can be calculated as follows.

$J_{p_{N}}=-q D_{p} \frac{d \Delta p_{n}\left(X_{n}\right)}{d x}=J_{n_{p_{0}}}+J_{0_{1 p}}\left(e^{\frac{V_{1}}{V_{T}}}-1\right)$

where

$$
\begin{aligned}
J_{n_{p_{0}}} & =\int_{\lambda_{\text {init }}}^{\lambda_{g}} \frac{-q L_{p}}{L_{p}^{2} \alpha^{2}-1} f(\lambda) \alpha(\lambda) e^{-\alpha\left(X_{n}+W_{n}\right)}\left\{\left[\frac{\left[\frac{D_{p}}{L_{p}} \sinh \left(\frac{W_{n}-X_{n}}{L_{p}}\right)-S_{P} \cosh \left(\frac{W_{n}-X_{n}}{L_{p}}\right)\right.}{S_{P} \sinh \left(\frac{W_{n}-x_{n}}{L_{p}}\right)-\frac{D_{p}}{L_{p}} \cosh \left(\frac{W_{n}-x_{n}}{L_{p}}\right)}\right]+L_{p} \alpha(\lambda)\right\} d \lambda \\
& -q \frac{D_{p}}{L_{p}}\left[\frac{D_{p} \frac{d \Delta p_{n}\left(G_{p}\left(W_{n}\right)\right)}{d x}-S_{P} \Delta p_{n}\left(G_{p}\left(W_{n}\right)\right)}{S_{P} \sinh \left(\frac{W_{n}-x_{n}}{L_{p}}\right)-\frac{D_{p}}{L_{p}} \cosh \left(\frac{W_{n}-x_{n}}{L_{p}}\right)}\right] \\
J_{0_{1_{p}}} & -q \frac{D_{p}}{L_{p}} p_{n_{0}}\left[\frac{\frac{D_{p}}{L_{p}} \sinh \left(\frac{W_{n}-X_{n}}{L_{p}}\right)-S_{P} \cosh \left(\frac{W_{n}-X_{n}}{L_{p}}\right)}{S_{P} \sinh \left(\frac{W_{n}-x_{n}}{L_{p}}\right)-\frac{D_{p}}{L_{p}} \cosh \left(\frac{W_{n}-x_{n}}{L_{p}}\right)}\right]
\end{aligned}
$$

Similarly, it can be done for minority electron carriers in the P region as follows.

$J_{n_{p}}=\left.q D_{n} \frac{d \Delta n_{p}(x)}{d x}\right|_{-X_{p}}=J_{p_{n_{0}}}+J_{0_{1_{n}}}\left(e^{\frac{V_{1}}{V_{T}}}-1\right)+J_{0_{2_{n}}}\left(e^{\frac{V_{2}}{V_{T}}}-1\right)$

where 


$$
\begin{aligned}
J_{p_{n_{0}}}= & \int_{\lambda_{\Delta}}^{\lambda_{g}} \frac{-2 q L_{n}}{L^{2}{ }_{n} \alpha^{2}-1} f(\lambda) \alpha(\lambda) e^{-\alpha\left(X_{p}-W_{p}\right)}\left\{\left[\frac{\left[\frac{D_{n}}{L_{n}} \sinh \left(\frac{-W_{p}+X_{p}}{L_{n}}\right)-S_{n} \cosh \left(\frac{-W_{p}+X_{p}}{L_{n}}\right)\right.}{S_{n} \sinh \left(\frac{-W_{p}+X_{p}}{L_{n}}\right)-\frac{D_{n}}{L_{n}} \cosh \left(\frac{-W_{p}+X_{p}}{L_{n}}\right)}\right]+L_{n} \alpha(\lambda)\right\} d \lambda \\
& +2 q \frac{D_{n}}{L_{n}}\left[\frac{D_{n} \frac{d \Delta n_{p}\left(G_{n}\left(-W_{p}\right)\right)}{d x}-S_{n} \Delta n_{p}\left(G_{n}\left(-W_{p}\right)\right)}{\left.S_{n} \sinh \left(\frac{-W_{p}+X_{p}}{L_{n}}\right)-\frac{D_{n}}{L_{n}} \cosh \left(\frac{-W_{p}+X_{p}}{L_{n}}\right)\right]}\right. \\
J_{0_{1_{n}}}= & q \frac{D_{n}}{L_{n}} n_{p_{0_{1}}}\left[\frac{\frac{D_{n}}{L_{n}} \sinh \left(\frac{-W_{p}+X}{L_{n}}\right)-S_{n} \cosh \left(\frac{-W_{p}+X_{p}}{L_{n}}\right)}{S_{n} \sinh \left(\frac{-W_{p}+X_{p}}{L_{n}}\right)-\frac{D_{n}}{L_{n}} \cosh \left(\frac{-W_{p}+X_{p}}{L_{n}}\right)}\right] \\
J_{0_{2_{n}}}= & q \frac{D_{n}}{L_{n}} n_{p_{0_{2}}}\left[\frac{D_{n} \sinh \left(\frac{-W_{p}+X}{L_{n}}\right)-S_{n} \cosh \left(\frac{-W_{p}+X_{p}}{L_{n}}\right)}{S_{n} \sinh \left(\frac{-W_{p}+X_{p}}{L_{n}}\right)-\frac{D_{n}}{L_{n}} \cosh \left(\frac{-W_{p}+X_{p}}{L_{n}}\right)}\right]
\end{aligned}
$$

To obtain the total current for the cell, the following relation can be used.

$$
J_{\text {Cell }}=J_{p_{n}}\left(-X_{p}\right)+J_{n_{1 p}}\left(-X_{p}\right)+J_{n_{2 p}}\left(-X_{p}\right)
$$

But, we need to calculate the minority hole current in the edge of the P-type semiconductor. It is easy to find it using the continuity equation in the intrinsic layer.

$J_{p_{n}}\left(-X_{p}\right)=J_{p_{n}}\left(X_{n}\right)-q \int_{-X_{p}}^{X_{n}} G(x) d x+q \int_{-X_{p}}^{X_{n}} R(x) d x$

It should mention that for the recombination rate in the intrinsic layer, we only consider the Schokley-Read-Hall mechanism.

$$
\begin{aligned}
J_{G_{C_{1}}} & =q \int_{-X_{p}}^{X_{n}} G(x) d x||_{\lambda_{\Delta}}^{\lambda_{g}}=q \int_{-X_{p}}^{X_{n}}\left[\int_{\lambda_{\Delta}}^{\lambda_{g}} f(\lambda) \cdot \alpha(\lambda) \cdot \mathrm{e}^{\left(-\alpha(\lambda)\left(x+w_{p}\right)\right)} d \lambda\right] d x \\
& =q \int_{\lambda_{\Delta}}^{\lambda_{g}} f(\lambda)\left[e^{-\alpha(\lambda)\left(W_{p}-X_{p}\right)}-e^{-\alpha(\lambda)\left(W_{p}+X_{n}\right)}\right] d \lambda
\end{aligned}
$$




$$
\begin{aligned}
J_{G_{C 2}} & =q \int_{-X_{p}}^{X_{n}} G(x) d x \mid \lambda_{\text {init }}=q \int_{-X_{p}}^{\lambda_{\Delta}}\left[\int_{\lambda_{\text {init }}}^{\lambda_{\Delta}} f(\lambda) \cdot \alpha(\lambda) \cdot \mathrm{e}^{\left(-\alpha(\lambda)\left(x+w_{p}\right)\right)} d \lambda\right] d x \\
& =q \int_{\lambda_{\text {init }}}^{\lambda_{A}} f(\lambda)\left[e^{-\alpha(\lambda)\left(W_{p}-X_{p}\right)}-e^{-\alpha(\lambda)\left(W_{p}+X_{n}\right)}\right] d \lambda
\end{aligned}
$$

The equation for the total flow in the conventional P-I-N junction as Eq. 27 is as follows.

$$
\begin{aligned}
J_{C e l l} & =J_{p_{n}}\left(-X_{p}\right)+J_{n_{1 p}}\left(-X_{p}\right)+J_{n_{2 p}}\left(-X_{p}\right) \\
& =\left[J_{n_{p_{0}}}+J_{0_{1 p}}\left(e^{\frac{V_{1}}{V_{T}}}-1\right)-J_{G_{C_{1}}}-J_{G_{C_{2}}}+J_{R_{0}}\left(e^{\frac{V_{1}}{2 K T}}-1\right)\right]+J_{p_{n_{0}}}+J_{0_{1_{n}}}\left(e^{\frac{V_{1}}{V_{T}}}-1\right)+J_{0_{2_{n}}}\left(e^{\frac{V_{2}}{V_{T}}}-1\right) \\
& =\left[J_{n_{p_{0}}}+J_{p_{n_{0}}}-J_{G_{C_{1}}}-J_{G_{C_{2}}}\right]+\left[\left(J_{0_{1_{n}}}+J_{0_{1 p}}\right)\left(e^{\frac{V_{1}}{V_{T}}}-1\right)\right]+\left[J_{R_{0}}\left(e^{\frac{V_{1}}{2 K T}}-1\right)\right]+\left[J_{0_{2_{n}}}\left(e^{\frac{V_{2}}{V_{T}}}-1\right)\right]
\end{aligned}
$$

Finally the power conversion efficiency can be obtained as follows.

$$
\eta=\frac{P_{M_{O_{1}}}+P_{M_{O 2}}}{P_{i n}}=\eta_{c_{1}}+\eta_{c_{2}}
$$

where $\eta_{c_{1}}$ and $\eta_{c_{2}}$ are power conversion efficiency related to contact 1 and contact 2 respectively.

\section{III-1. Heat Generation in single contact solar cell:}

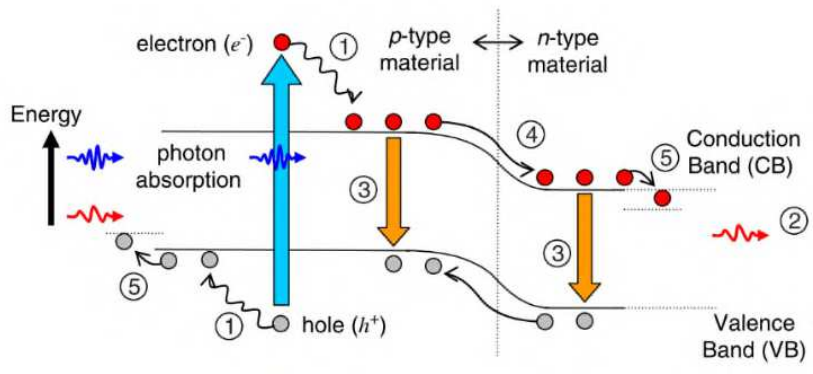

Fig. 3. Loss mechanism in a single junction solar cell

For a single-junction solar cell, (Fig. 3) the extra energy of hot electrons through electron-electron and electronlattice scattering is transferred to heat. In the following, the difference between a single contact and two selective energy contacts solar cells, from a heat generation point of view, will be compared. Also, the authors will be focusing on the power conversion efficiency degradation. For single junction and single contact cell the generated heat is given as follows. 
$H_{\text {S.J.S.C }}=\int_{E_{C}}^{\infty}\left(E-E_{C}\right) D(E) F(E) d E$

For single junction and two selective energy contacts, heat generation is calculated as follows.

$$
\begin{aligned}
H_{\text {S.J.T.C }} & =\int_{E_{C}}^{E_{C}+\Delta E}\left(E-E_{C}\right) D(E) F(E) d E+\int_{E_{C}+\Delta E}^{\infty}\left(E-E_{C}-\Delta E\right) D(E) F(E) d E \\
& =\int_{E_{C}}^{\infty}\left(E-E_{C}\right) D(E) F(E) d E-\Delta E \int_{E_{C}+\Delta E}^{\infty} D(E) F(E) d E
\end{aligned}
$$

By comparing the two cases, the difference between generated heat is as follows.

$$
H_{\text {S.J.T.C }}=H_{\text {S.J.S.C }}-\Delta E \int_{E_{C}+\Delta E}^{\infty} D(E) F(E) d E
$$

Thus, using two contacts, the operating temperature decreases as follows.

$$
\Delta E_{\text {Thermal }}=\Delta E \int_{E_{C}+\Delta E}^{\infty} D(E) F(E) d E
$$

If one likes to calculated how temperature is changed, the following relation can be used.

$T_{O_{\text {P.J.T.C }}}=T_{\text {S.J.S.C }}-\frac{2}{3 K_{B}} \Delta E \int_{E_{C}+\Delta E}^{\infty} D(E) F(E) d E$

In general for more than two contacts the thermal energy decreasing can be calculated as follows.

$$
\begin{aligned}
\Delta E_{\text {Thermal }} & =\Delta E_{1} \int_{E_{c}+\Delta E_{1}}^{\infty} D(E) F(E) d E+\Delta E_{2} \int_{E_{c}+\Delta E_{1}+\Delta E_{2}}^{\infty} D(E) F(E) d E+\Delta E_{3} \int_{E_{c}+\Delta E_{1}+\Delta E_{2}+\Delta E_{3}}^{\infty} D(E) F(E) d E+\ldots \\
& =\sum_{i=1}^{M} \Delta E_{i} \int_{E_{c}+\sum_{m=1}^{\infty} \Delta E_{m}}^{\infty} D(E) F(E) d E
\end{aligned}
$$

where $\mathrm{M}$ is the number of contacts above conduction band, $\Delta E_{i}$ is the energy difference between contacts $i+1$ and i. This is the contribution due to electron scattering energy loss, and also similar to this calculation can be done for hole carrier. Thus by using selective energy contacts high-efficiency power conversion can be achieved and operating temperature can be controlled and decreased as well.

\section{IV: Simulation Results}


In this section, first of all, we examine the results when the single contact is used on the solar panel on the $p$ side and then the results will be compared with the two-contact mode. It is shown that in the case of two contacts, the efficiency of the power conversion is enhanced. Fig. 4 shows results of the I-V curve and efficiency of single and two contacts cases.

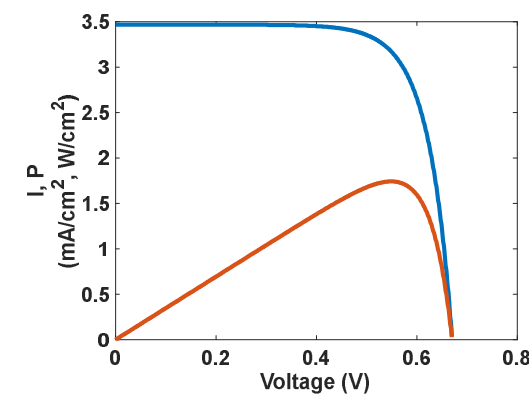

(a)

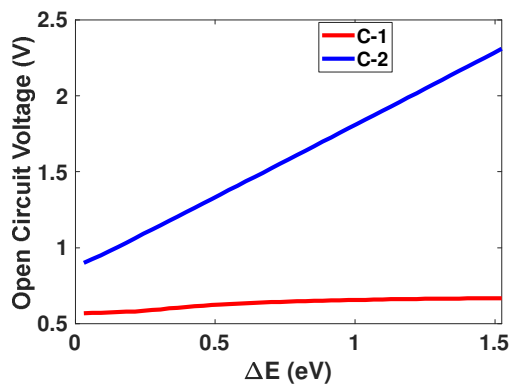

(d)

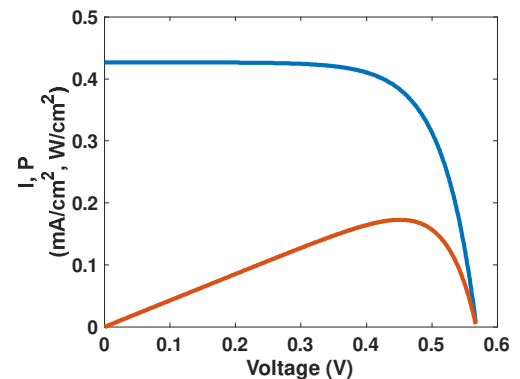

(b)

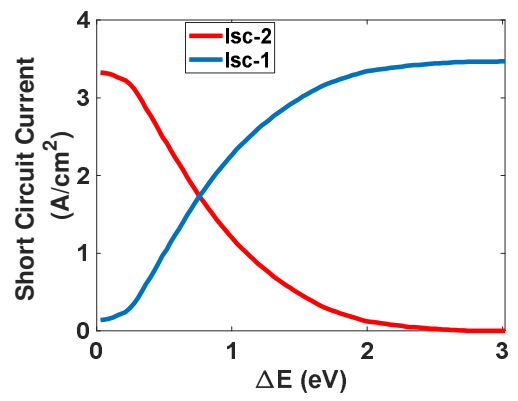

(e)

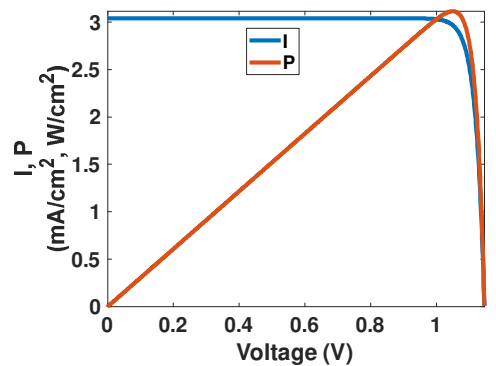

(c)

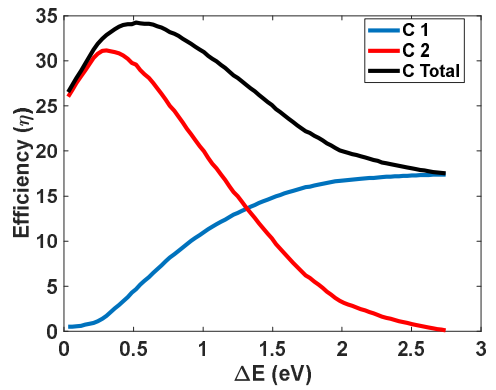

(f)

Fig. 4. a) I-V, P curve of single contact PIN solar cell, b, c) I-V, P curve of two contacts (C-1, C-2) PIN solar cell for $\Delta E=0.3$ eV, d) Open circuit properties of contacts 1 and contact 2 in terms of increasing $\Delta E$ e) Short circuit properties of contacts 1 and contact 2 in terms of increasing $\Delta E$ f) Characteristics of efficiencies enhancement in terms of $\Delta E$

Figure 4 shows the current-voltage (I-V) and power-voltage (P-V) characteristics of a perovskite solar cell with the thickness and characteristics given in Table 1. As explained in the previous section, we will have different responses according to the continuity equations as well as the generation rate energy interval for each contact according to the selected $\Delta E$ s. This means that we locate the contacts at a distance of $\Delta E$ from the conduction band to infinity, during which we obtain the maximum and optimal conversion efficiency at a certain point. This operation continues until all the carriers are gathered in one contact and practically no efficiency is taken from the second contact. Naturally, in the meantime, the output efficiencies of contacts 1 and 2 affect each other. This is, perhaps, because the carriers in each band are independent of each other. Finally, by adding the two output conversion efficiencies of each contact, the overall efficiency of the cell is proportional to $\Delta \mathrm{E}$ or the energy distance from the conduction band. As the $\Delta \mathrm{E}$ changes or the distance from the conduction band rises, the current-voltage and power-voltage characteristics are changed. Fig. 
4 shows the open-circuit voltage, short-circuit current, and efficiency separately. It can be seen, from Fig. 4 , that the efficiency is optimum for a given $\Delta \mathrm{E}$ in the two contacts case, and in general, there is an optimum $\Delta \mathrm{E}$ vector for contacts to obtain maximum efficiency. Also, it can be noticed, from Fig. 4, that the open-circuit voltage for contact-2 is increased with increasing $\Delta \mathrm{E}$ and for contact-1 it is increased but limited to diode built-in voltage. It is well known that with increasing $\Delta \mathrm{E}$ open circuit voltage for contact-2 must be increased. Similarly, the short circuit current for contact- 2 with increasing $\Delta \mathrm{E}$ is decreased because of decreasing the electron density. It is inverse for contact-1 and with increasing $\Delta \mathrm{E}$ the density of electrons is increased and so the short circuit current is increased excessively. Additionally, considering the simulated results, the efficiency in the first contact is initially lower (for $\Delta \mathrm{E}=0$ ) and gradually increases to saturate the final value that is equal to the standard single contact solar cell. In the second contact for $\Delta E=0$, the efficiency is maximum and with increasing $\Delta \mathrm{E}$ the efficiency is decreased because the density of electrons is approaching zero. The total power conversion efficiency is enhanced with enhancing $\Delta \mathrm{E}$, and it reaches the maximum value that is larger than the single contact case and then with enhancing more $\Delta E$, the total efficiency is decreased to the efficiency of a single contact case.

Now, after evaluation of the proposed solar cell's performance, we can calculate the heat generation and how multi selective energy contacts can minimize the heat generation. Based on the mathematical modeling presented in Eqs. 3338 , in the following for two selective energy contacts, the density of electrons and heat reduction versus energy difference between contacts are presented in Fig. 5 a and b. It can be seen, from Fig. 5, that the density of electrons for the second contact is decreased with enhancing the energy difference and the inverse situation is for the first contact. Also, reduction of the heat generation has a maximum and for zero and infinity energy differences the reduction of heat generation are going to be zero.

For three contacts reduction of generated heat is illustrated in Fig. 5 c. It is clear that in this case there are optimum values for both energy differences.

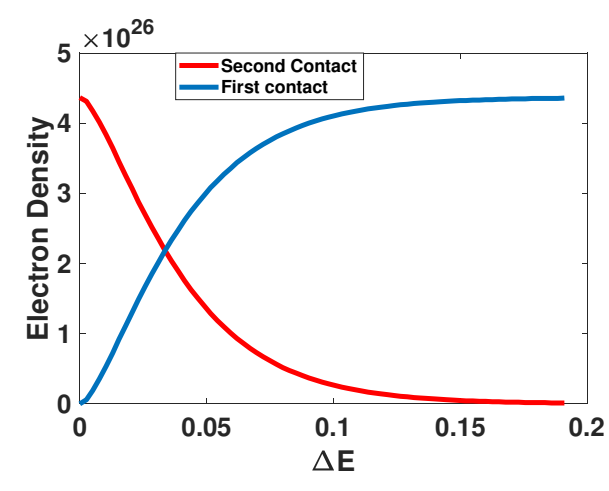

a

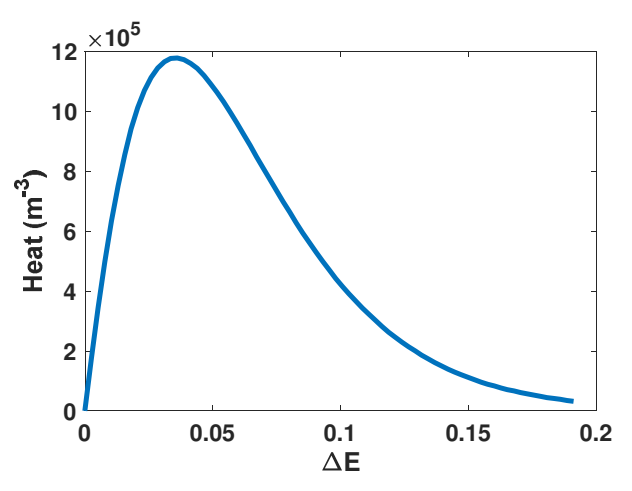

b 


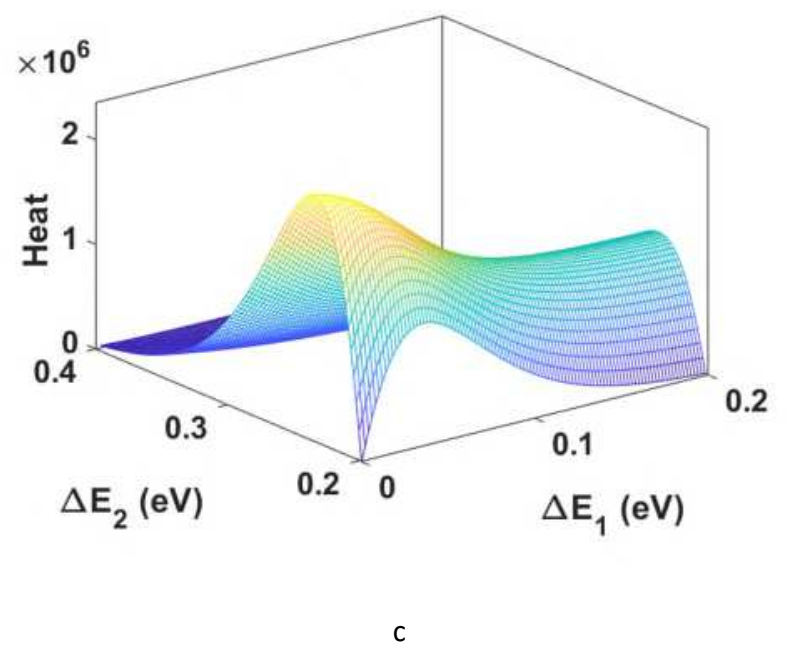

Fig. 5. A) Electron density at contacts $\left(\mathrm{m}^{-3}\right)$, B) Reduction of Heat generated in two contacts solar cell $\left(\mathrm{J} / \mathrm{m}^{3}\right)$ and C) Reduction of Heat generated in three contacts solar cell $\left(\mathrm{J} / \mathrm{m}^{3}\right)$

\section{Conclusion}

There is wide-ranging support worldwide for the use of renewable energy, especially solar energy, which offers electricity without causing any carbon dioxide releases. Accordingly, the main goal is to reduce the solar cells manufacturing costs and also increasing power conversion efficiency. When we take the Carnot limit into our consideration, which is a relatively high number, it turns out that there are still many problems in obtaining highefficiency solar cells. Therefore, this study was originally designed to provide a suitable simulation of high-efficiency, affordable and easy-to-install solar cells. To reduce the price, perovskite material has been used, which has lower manufacturing and processing cost compared to silicon and other materials used in the solar cell industry. Examining the optical and electrical properties of this material, this study shows that due to its excellent light absorption, the conversion efficiency of solar cells can be increased dramatically. The energy selective contacts method was utilized successfully in this study to increase the efficiency, reduce heat loss and death of high-energy carriers. Therefore, this method is a suitable way to prevent solar panels from overheating. Finally, this study shows that when two contactors are used, the efficiency is greatly increased and the heat generated is reduced ensuring long-life operation.

\section{References}

[1] H.-S. Kim, C.-R. Lee, J.-H. Im, K.-B. Lee, T. Moehl, A. Marchioro, S.-J. Moon, R. Humphry-Baker, J.-H. Yum, J. E. Moser, M. Grätzel, N.-G. Park, Sci. Rep. 2012, 2, 591.

[2] M. M. Lee, J. Teuscher, T. Miyasaka, T. N. Murakami and H. J. Snaith, Science, 2012, 338, 643.

[3] M. M. Lee, J. Teuscher, T. Miyasaka, T. N. Murakami and H. J. Snaith, Science, 2012, 338, 643.

[4] H. S. Kim, C. R. Lee, J. H. Im, K. B. Lee, T. Moehl, A. Marchioro, S. J. Moon, R. Humphry-Baker, J. H. Yum, J. E. Moser, M. Gr“atzel and N. G. Park, Sci. Rep., 2012, 2, 591 
[5] M. M. Lee, J. Teuscher, T. Miyasaka, T. N. Murakami, H. J. Snaith,Science 2012, 338, 643.

[6] Q. Dong, Y. Fang, Y. Shao, P. Mulligan, J. Qiu, L. Cao and J. Huang, Solar cells. Electron-hole diffusion lengths $>175$ mum in solution-grown CH3NH3PbI3 single crystals, Science, 2015, 347, 967-970.

[7] M. Liu, M. B. Johnston, and H. J. J. N. Snaith, Efficient planar heterojunction perovskite solar cells by vapor deposition, Nature, 2013, 501, 395-398.

[8] J. H. Heo, S. H. Im, J. H. Noh, T. N. Mandal, C. Lim, J. A. Chang, Y. H. Lee, H. Kim, A. Sarkar, M. K.Nazeeruddin, M. Graetzel, and S. I. Seok, Nat. Photonics 7, 487 (2013).

[9] S. D. Stranks, G. E. Eperon, G. Grancini, C. Menelaou, M. J. P. Alcocer, T. Leijtens, L. M. Herz, A. Petrozza,and H. J. Snaith, Science 342, 341 (2013).

[10] G. Xing, N. Mathews, S. Sun, S. S. Lim, Y. M. Lam, M. Graetzel, S. Mhaisalkar, and T. C. Sum, Science 342, 344 (2013).

[11] A. Kojima, K. Teshima, Y. Shirai, and T. Miyasaka, Organometal halide perovskites as visible-light sensitizers for photovoltaic cells, J. Am. Chem. Soc., 2009, 131, 6050-6051.

[12]National Renewable Energy Laboratory, Best research-cell efciencies,2019,www.nrel.gov/pv/assets/pdfs/pv-efciencychart. 20190103.pdf.

[13] Koh, T. M.; Fu, K.; Fang, Y.; Chen, S.; Sum, T. C.; Mathews, N.; Mhaisalkar, S. G.; Boix, P. P.; Baikie, T. FormamidiniumContaining Metal-Halide: An Alternative Material for Near-IR Absorption Perovskite Solar Cells. J. Phys. Chem. C 2014, 118, 16458-16462.

[14] Kim, H.-S.; Lee, C.-R.; Im, J.-H.; Lee, K.-B.; Moehl, T.; Marchioro, A.; Moon, S.-J.; Humphry-Baker, R.; Yum, J.-H.; Moser, J. E.; Grätzel, M.; Park, N.-G. Lead lodide Perovskite Sensitized All-Solid-State Submicron Thin Film Mesoscopic Solar Cell with Efficiency Exceeding 9\%. Sci. Rep. 2012, 2, 591.

[15] Hao, F.; Stoumpos, C. C.; Cao, D. H.; Chang, R. P. H.; Kanatzidis, M. G. Lead-Free Solid-State Organic-Inorganic Halide Perovskite Solar Cells. Nat. Photonics 2014, 8, 489-494.

[16] W. S. Yang, B. W. Park, E. H. Jung, Jeon, Nam Joong, Y. C. Kim, D. K. Lee, S. S. Shin, J. Seo, E. K. Kim, J. H. Noh, S. Seok, Science 356, 1376 (2017).

[17] a) J. W. Xiao, L. Liu, D. Zhang, N. De Marco, J. W. Lee, O. Lin, Q. Chen, Y. Yang, Adv. Energy Mater. 2017, 7, 1700491; b) F. Bella,P. Renzi, C. Cavallo, C. Gerbaldi, Chem. - Eur. J. 2018, 24, 12183.

[18] S. Shayan, S. Matloub, and A. Rostami, "Efficiency enhancement in a single bandgap silicon solar cell considering hot-carrier extraction using selective energy contacts," Optics Express, Vol. 29, No. 4, 2021.

[19] Hui-Jing Du, Wei-Chao Wang, Yi-Fan Gu Simulation design of P-I-N-type all-perovskite solar cells with high efficiency 2017 Chin. Phys. B 26028803.

[20] Todorov, T., O. Gunawan, and S. Guha. "A road towards 25\% efficiency and beyond: perovskite tandem solar cells." Molecular Systems Design \& Engineering 1.4 (2016): 370-376.

[21] Niu, Guangda, et al. "Enhancement of thermal stability for perovskite solar cells through cesium doping," RSC advances 7.28 (2017): 17473-17479. 